COMMENT. Working memory of long-term survivors of childhood cerebellar tumors is not affected following surgical resection, provided they have not received radio- or chemotherapy. In contrast, postural imbalance and incoordination are sustained when deep cerebellar nuclei have been involved, and the failure to recover motor function postsurgically is not dependent on the age of the patient or the size of the lesion. The site of the lesion, specifically involvement of the nuclei fastigius, interpositus, and dentatus, is correlated with incomplete recovery of motor function following cerebellar tumor resection. The authors of the above study comment that a more comprehensive battery of tests may have uncovered cognitive deficits in their patients.

Long-term cognitive sequelae after cerebellar astrocytoma surgery, without additional radio- and chemotherapy, were reported in a series of 23 children studied in the Netherlands (Aarsen FK et al. Neurology 2004;62:1311-1316; Ped Neur Briefs 2004;18:39-40). Neuropsychological testing at 1-8 years after tumor resection and 6-22 years of age showed significantly weaker performances in sustained attention, executive functioning, visualspatial function and memory. A high percentage required special education, reflecting the severity and persistence of cognitive deficits.

The cerebellum has 3 functional zones, medial, intermediate, and lateral (Jansen, Brodal, 1940). The medial zone contains the vermis and nuclei fastigii, concerned with control of posture and locomotion. Damage to the intermediate zone, containing the nucleus interpositus and dorsomedial dentate nucleus, causes tremor. The anterior portion of the lateral cerebellar hemisphere and the dentate nucleus are involved in motor control, while the posterolateral portion governs motor planning, language development and memory function. In the current Minnesota study, one third of the tumors were medulloblastomas, and the lateral hemispheres concerned with cognitive function were less involved than vermal structures.

\title{
EEG AND MRI IN IDENTIFYING TUBEROUS SCLEROSIS LESIONS FOR EPILEPSY SURGERY
}

The role of surface EEG and MRI in the identification of epileptogenic tubers for surgical resection in patients with tuberous sclerosis (TSC) was studied at the Cleveland Clinic, OH. Localized and concordant preoperative MRI and EEG abnormalities were found in 9 of 17 patients (median age 12 years; range 2 months to 31 years), and resection of a localized tuber provided seizure freedom in $8(89 \%)$ patients. Of 8 patients with less welllocalized or non-concordant MRI and EEG, only $3(38 \%)$ were seizure free and 5 had persistent seizures following surgery. Overall, $11 / 17(65 \%)$ patients became seizure free following surgery. The response was not correlated with age at time of surgery or type of surgery (temporal resection vs frontal/multilobar resections). TSC is not a contraindication to epilepsy surgery. (Lachhwani DK, Pestana E, Gupta A et al. Identification of candidates for epilepsy surgery in patients with tuberous sclerosis. Neurology May (1 of 2) 2005;64:16511654). (Reprints: Dr Deepak K Lachhwani, Section of Pediatric Epilepsy and Pediatric Neurology, The Cleveland Clinic Foundation, 9500 Euclid Ave, S-51, Cleveland, OH 44195).

COMMENT. Surface EEG and MRI are effective in localizing epileptogenic tubers for epilepsy surgery in tuberous sclerosis. Patients with concordant EEG and MRI 
abnormalities have optimal seizure control following surgical resection of the localized tuber. The localization of TSC lesions not showing concordant findings on MRI and EEG requires presurgical testing by surface video-EEG, PET, ictal SPECT, diffusion-weighted MRI, or intraoperative ECoG (Romanelli P et al. Pediatr Neurol 2004;31:239-247; Ped Neur Briefs 2004;18:77-78). In this previous study, resection of tubers and epileptogenic foci localized by the above methods resulted in control of seizures in $78 \%$ and reduction of seizure frequency in $20 \%$. Early detection and resection of refractory seizure foci can prevent deterioration of cognitive functioning, improve behavior, and lead to a better quality of life.

\section{NEUROPATHIES}

\section{SUBACUTE DEMYELINATING POLYNEUROPATHY}

The electroclinical characteristics of 5 children (ages 4-13 years) with subacute inflammatory demyelinating polyneuropathy (SIDP) are reported from the Royal Children's Hospital, Victoria, Australia. Onset followed 2-13 days after a nonspecific infection with fever, upper respiratory tract symptoms or gastroenteritis in 3 patients; one had recent cytomegalovirus infection; none had Campylobacter jejuni. All had leg weakness (with pain in 3) at presentation, and upper limb involvement followed. All remained ambulant. Neurologic findings included areflexia in 5, ataxia in 4, and bilateral facial weakness in 1 . CSF protein was elevated $(0.75-1.5 \mathrm{~g} / \mathrm{L})$ in 3 , and leukocytes were absent or $1 / \mathrm{mm}^{3}$. Nerve conduction studies were abnormal. Period from onset of symptoms to treatment was 4-8 weeks. All received oral prednisolone for 1.5-6 months, and all showed improved muscle strength within 1 week. Three had returned to normal within 6 months and one within 8 months, and none showed relapse at 6-20 years follow-up. One was normal when last seen at 10 months follow-up. (Rodriguez-Casero MV, Shield LK, Kornberg AJ. Subacute inflammatory demyelinating polyneuropathy in children. Neurology May (2 of 2) 2005;64:1786-1788). (Dr AJ Kornberg, Department of Neurology, Royal Children's Hospital, Flemington Rd, Parkville, Victoria, Australia 3052).

COMMENT. The authors have differentiated this series of SIDP patients with a benign, subacute illness and monophasic course of 4-8 weeks from the acute form of inflammatory demyelinating polyneuropathy (Guillain-Barre syndrome [GBS]) witì à 4 week course, and a chronic form (CIDP) with progression over 8 weeks and frequent relapse. SIDP characteristics that differ from GBS include 1) longer period of progression; 2) lack of respiratory, cranial nerve, or autonomic involvement; 3 ) more abnormal nerve conduction in initial stages; and 4) rapid and sustained response to corticosteroids. The monophasic and nonrelapsing course of SIDP differentiates it from CIDP. The response of CIDP to steroids is reviewed in Ped Neur Briefs 2005;19:19).

\section{EARLY ONSET CHARCOT-MARIE-TOOTH DISEASE}

The clinical signs and genetic analysis of early-onset Charcot-Marie-Tooth disease (CMT) in a 2-year-old boy and members of his family are reported from the Academic Medical Center, Amsterdam, and Sophia Children's Hospital, Rotterdam, the Netherlands. 